\title{
A Coordinate Descent Algorithm for Multichannel Multiuser Detection in Underwater Acoustic DS-CDMA Systems
}

\author{
Eduard Calvo Page ${ }^{1}$ \\ Signal Processing and Communications Group \\ Technical University of Catalonia (UPC) \\ Jordi Girona 1-3, Campus Nord, Edifici D5, \\ 08034 Barcelona (SPAIN) \\ Email: eduard@gps.tsc.upc.edu
}

\author{
Milica Stojanovic \\ Sea Grant College Program \\ Massachusetts Institute of Technology (MIT) \\ Bldg. E38-376 \\ Cambridge, MA 02139 (USA) \\ Email: millitsa@mit.edu
}

\begin{abstract}
Direct Sequence (DS) Code Division Multiple Access (CDMA) is considered for a network of Autonomous Underwater Vehicles (AUVs) communicating through a Base Station (BS) equipped with a receive array. A multiuser detection algorithm, based on the Cyclic Coordinate Descent (CCD) approach is proposed for this situation. The CCD technique is used to circumvent the complexity of the optimal Maximum Likelihood (ML) detector. The proposed algorithm is based on joint data detection and channel estimation using Minimum Mean Square Error (MMSE) as the overall optimization criterion. It is implemented in a multichannel configuration to exploit the array processing gain available at the BS. The complexity of the proposed CCD detector does not depend on the modulation level and is linear in the size of the receive array. The CCD detector has been tested using real data, recorded from four users transmitting asynchronously over a shallow water acoustic channel, with varying Spreading Factors. The signals were transmitted over $2 \mathrm{~km}$ in a $20 \mathrm{kHz}$ band, demonstrating good results at the chip rate of $16 \mathrm{kchips} / \mathrm{second}$.
\end{abstract}

\section{INTRODUCTION}

Advanced search and rescue missions require a network of multiple AUVs to be deployed in a footprint of a few kilometers around a BS. The communication channel can be shared among the users through frequency, time, or code-division multiple access (TDMA, FDMA, CDMA). The choice of DSCDMA eliminates strict requirements for frame scheduling and provides the advantages of multipath resolution and robustness to interference [1].

When designing algorithms for detection of multiple users' signals, two approaches can be taken: single-user detection, or multiuser detection. In the first approach, a bank of singleuser detectors, one for each user, is implemented, and each detector treats the undesired signals (interferers) as noise. This approach yields computationally simple receivers, but it does not exploit the knowledge about the structure of interference, although the BS possesses all the users' code sequences. In contrast, a multiuser detector (MUD) uses knowledge of all

\footnotetext{
${ }^{1}$ This work was done during an internship at the Sea Grant College Program, MIT, in 2004.
}

the code sequences to suppress interference and perform joint detection of all the users' signals. Although more complex, this approach offers performance gain in scenarios with high levels of interference [2].

In an underwater acoustic environment, asynchronous transmission, extended multipath propagation, fast variation of the channel impulse response, and Doppler distortion result in the loss of signal orthogonality, requiring a sophisticated detection algorithm to overcome the channel impairments. The proposed MUD algorithm performs joint MMSE channel estimation and data detection, suitable for such highly distorted channels. It is derived from the optimal ML detector, but unlike this detector which must test all possible data sequences, and hence has prohibitive complexity, the proposed algorithm is based on Cyclic Coordinate Descent (CCD), and has complexity that is linear in the number of users.

The signal model is outlined in Section II, while the algorithm operation and its multichannel implementation are addressed in Section III. The performance of the CCD detector is illustrated using experimental data in Section IV. Finally, Section V concludes the paper.

\section{SignAl MODEL}

Let us assume that the total number of users is $K$ and that the spreading factor used by all of them is SF (we do not consider multirate transmissions in the network). $c_{k}(t)=$ $\sum_{i=0}^{\mathrm{SF}-1} c_{k}[i] p_{c}\left(t-i T_{c}\right)$ denotes one period of the code of the $k-t h$ user. The chip shaping pulse, $p_{c}(t)$, is taken as squared root raised cosine, and the overall channel as seen by the $k-t h$ user, $h_{k}(t)$, is assumed to have a delay spread less than the symbol interval $T=\operatorname{SF} T_{c}$ ( $T_{c}$ stands for the chip duration). The sampling rate at the receiver is twice the chip rate, i.e., $N_{s c}=2$ samples per chip are used. It is useful to define the indices of symbols, chips and samples in order to ease the notation for further analysis:

- $m$ - current sample index; $m \in\left\{0,1, \ldots \ldots, N_{d} S F N_{s c}-\right.$

1) ( $N_{d}$ is the total number of transmitted data symbols)

- $r$ - current symbol index; $r \in\left\{0,1, \ldots, N_{d}-1\right\}$ 
- $i$ - chip index within the current symbol; $i \in$ $\{0,1, \ldots, \mathrm{SF}-1\}$

- $j$ - sample index within the current chip of the current symbol; $j \in\left\{0,1, \ldots, N_{s c}-1\right\}$

The relation between these indices is

$$
m=\mathrm{SF} N_{s c} r+N_{s c} i+j .
$$

The transmitted signal of the $k$-th user is

$$
u_{k}(t)=\sum_{r=-\infty}^{+\infty} d_{k}[r] c_{k}(t-r T)
$$

and the signal at the receiver, $v(t)$, is given by

$$
v(t)=\sum_{k=1}^{K} v_{k}(t)+n(t)=\sum_{k=1}^{K} h_{k}(t) * u_{k}(t) e^{j \theta_{k}(t)}+n(t),
$$

where $\left\{\theta_{k}(t)\right\}_{k=1 \ldots K}$ are phase distortion terms and $n(t)$ is noise. The equivalent discrete-time model is

$v[m]=\sum_{k=1}^{K} v_{k}[m]+n[m]=\sum_{k=1}^{K} \mathbf{h}_{k}^{T}[m] \mathbf{u}_{k}[m] e^{j \theta_{k}[m]}+n[m]$.

The block diagram of the system is shown in Figure 1.

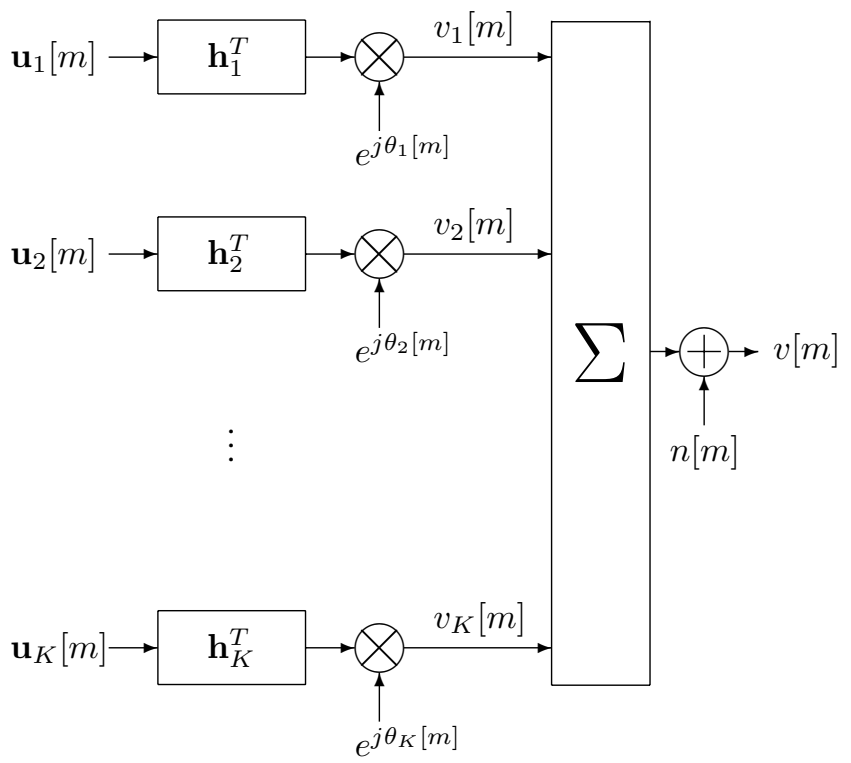

Fig. 1. Block diagram of the system.

The channel vectors $\left\{\mathbf{h}_{k}[m]\right\}_{k=1 \ldots K}$ and the transmitted signal vectors $\left\{\mathbf{u}_{k}[m]\right\}_{k=1 \ldots K}$ have a time span of $T$ seconds (SF $N_{s c}$ samples). The latter is of the form

$$
\mathbf{u}_{k}[m]=\left[d_{k}[r] \mathbf{c}_{k}^{r T}[m] \quad d_{k}[r-1] \mathbf{c}_{k}^{r-1^{T}}[m]\right]^{T} .
$$

While $\mathbf{c}_{k}$ is a vector containing all the SF chips of the code of the $k$-th user repeated $N_{s c}$ times each (because of sampling), $\mathbf{c}_{k}[m]$ is a circularly shifted $\left(N_{s c} i+j\right.$ samples) version of $\mathbf{c}_{k}$, as illustrated in Figure 2. It can be split into the contribution of the current $\left(\mathbf{c}_{k}^{r}[m]\right)$ and the past $\left(\mathbf{c}_{k}^{r-1}[m]\right)$ symbol, so that
$\mathbf{c}_{k}[m]=\left[\mathbf{c}_{k}^{r}[m]^{T} \mathbf{c}_{k}^{r-1}[m]^{T}\right]^{T}$. Assuming that the last data symbol is known, $\mathbf{u}_{k}[\mathrm{~m}]$ can be reconstructed at the receiver up to the value of $d_{k}[r]$.

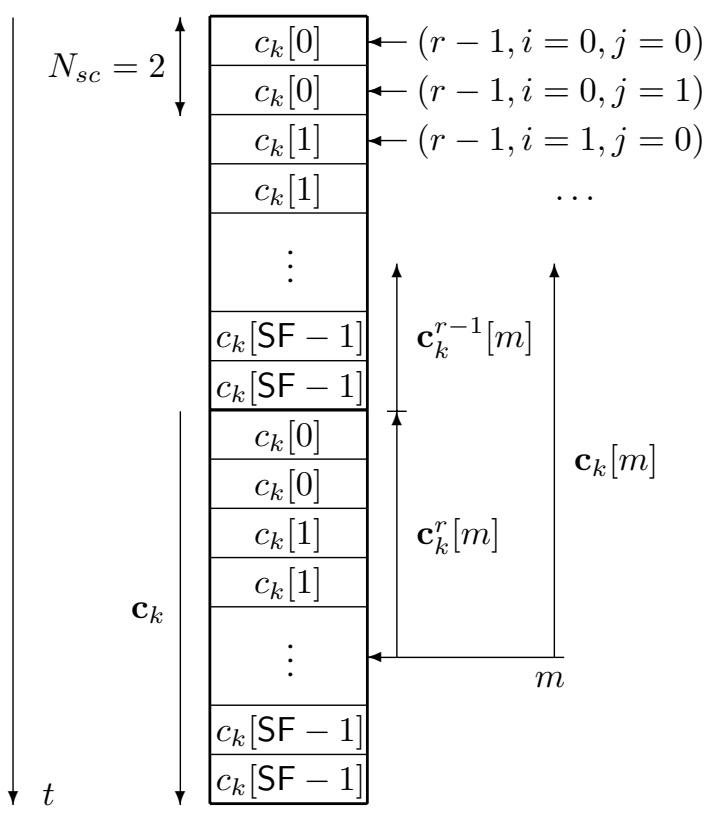

Fig. 2. Structure of the vectors $\mathbf{c}_{k}, \mathbf{c}_{k}[m]$.

\section{THE CCD DETECTOR}

\section{A. The single-channel CCD Detector}

The task of the detector is to extract the data symbols $\mathbf{d}=\left[d_{1} \ldots d_{K}\right]^{T}$ from the received signal $v[m]$ (4), which is distorted by the channels $\left\{\mathbf{h}_{k}\right\}_{k=1}^{K}$ and the phase terms $\left\{e^{j \theta_{k}[m]}\right\}_{k=1}^{K}$. We assume without loss of generality that by the time the $m$-th sample of the $r$-th symbol interval is received, we have an estimate $\hat{\mathbf{d}}[r, m]=\left[\hat{d}_{1}[r, m] \ldots \hat{d}_{K}[r, m]\right]^{T}$ of the vector of transmitted symbols, as well as reliable estimates of the phase terms and the channel responses, denoted by $\left\{e^{j \hat{\theta}_{k}[m]}\right\}_{k=1}^{K}$ and $\left\{\mathbf{a}_{k}^{*}[m]\right\}_{k=1}^{K}$, respectively. The received signal $v[m]$ can then be estimated at the BS as

$$
\hat{v}[m]=\sum_{k=1}^{K} \mathbf{a}_{k}^{H}[m] \hat{\mathbf{u}}_{k}[m] e^{j \hat{\theta}_{k}[m]}
$$

where the set of vectors $\left\{\hat{\mathbf{u}}_{k}\right\}_{k=1}^{K}$ are the reconstructed transmitted vectors obtained from (5) using $\left\{\hat{d}_{k}[r, m]\right\}_{k=1}^{K}$ instead of $\left\{d_{k}[r]\right\}_{k=1}^{K}$ and the decisions $\left\{\widetilde{d}_{k}[r-1]\right\}_{k=1}^{K}$ instead of $\left\{d_{k}[r-1]\right\}_{k=1}^{K}$. This estimate produces the error

$$
e[m]=v[m]-\hat{v}[m]
$$

which is used to update the estimates of symbols, phases, and channels, according to the MMSE criterion. The structure of the detector is shown in Figure 3.

The MSE is a multivariate cost function that depends on the estimates of symbols, phases, and channels. However, the error (7) does not exhibit linear dependence on a vector containing all of these variables (the MSE is not a quadratic form of it). 


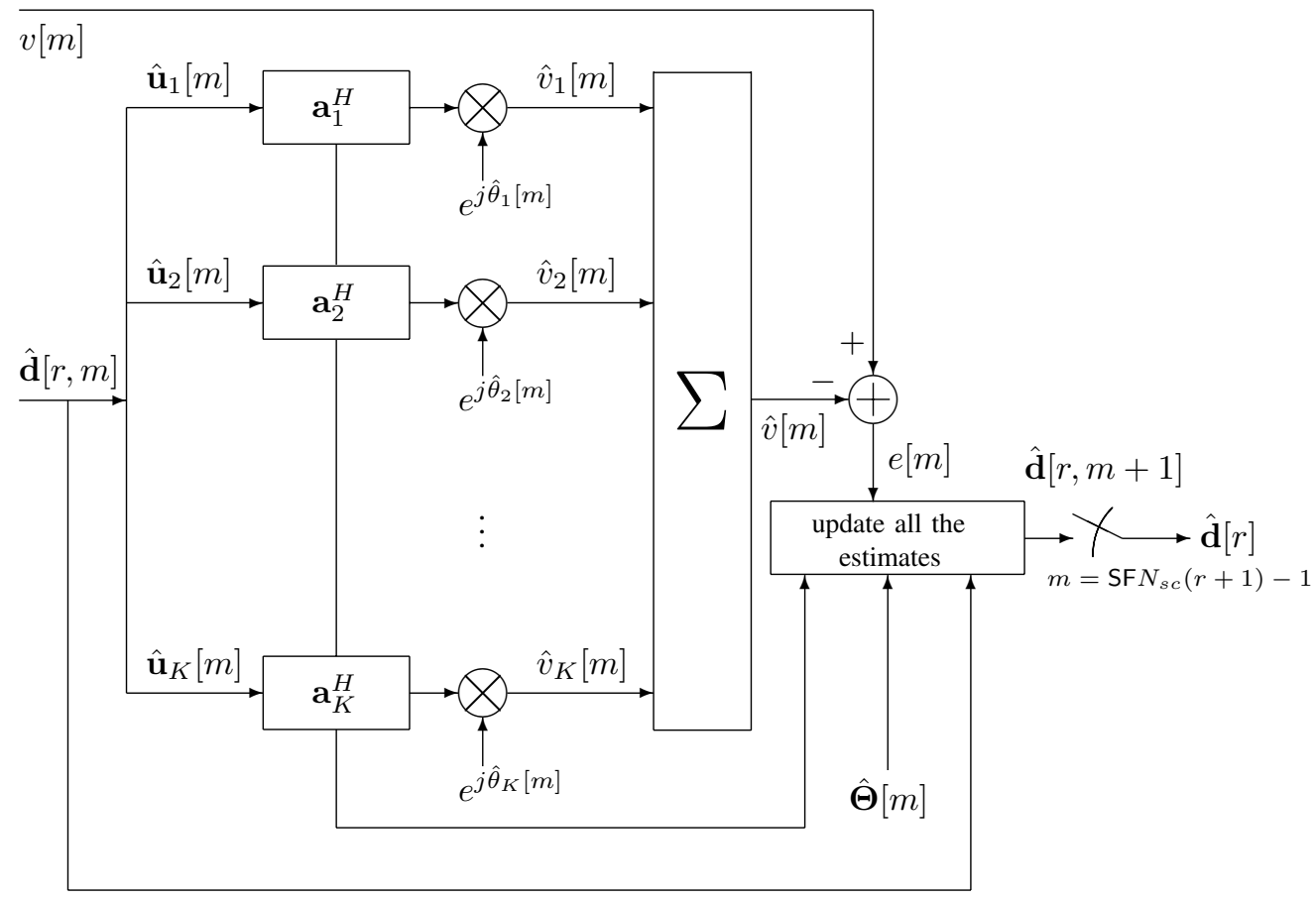

Fig. 3. Block diagram of the single-channel CCD Detector.

Hence, standard adaptive algorithms (LMS, RLS) cannot be directly applied to perform joint update of the entire set of variables.

CCD algorithms can be used to minimize multivariate cost functions that are not quadratic in the vector containing all the variables but are quadratic in each variable given the rest. They have been used in many areas such as tomography, inverse kinematics, or bioinformatics. They have also been applied to multi-user detection for synchronous CDMA [3] [4]. A CCD algorithm performs in a definite (cyclic) order the optimization of each variable separately given the rest of them. Thus, an iteration of a CCD algorithm consists of one-by-one optimization procedures for all the variables in a given order, starting from proper initial values. For the problem of MMSE MUD, we identify three different variables: a vector containing the vertically stacked estimated response of the channels of all the users $\left(\mathbf{a}[m]=\left[\mathbf{a}_{1}^{T}[m] \ldots \mathbf{a}_{K}^{T}[m]\right]^{T}\right)$, a vector containing the estimates of the users' phase terms $\left(\hat{\boldsymbol{\Theta}}[m]=\left[e^{j \hat{\theta}_{1}[m]} \ldots e^{j \hat{\theta}_{K}[m]}\right]^{T}\right)$, and the vector of symbol estimates $(\hat{\mathbf{d}}[r, m])$.

The data packets transmitted by the users consist of a training preamble plus the data sequence. We assume that after the training period reliable estimates of channels $\mathbf{a}[\mathrm{m}]$ and phases $\hat{\boldsymbol{\Theta}}[m]$ are available. The samples of the received signal $v[m]$ corresponding to the $r$-th symbol interval are collected at Nyquist's rate $\left(N_{s c}=2\right)$ in a block and an all-zeros initial value of the symbol estimates is assumed.

Given $\hat{\boldsymbol{\Theta}}[m]$ and $\mathbf{a}[m]$, the error can be shown to be linear in the symbols' estimates as

$$
e[m]=v[m]-v^{r-1}[m]-\hat{\mathbf{d}}[r, m]^{T} \mathbf{b}[m],
$$

where $v^{r-1}[m]$ contains the contribution of the latest decisions

$$
v^{r-1}[m]=\sum_{k=1}^{K} \mathbf{a}_{k}^{r-1 H} \mathbf{c}_{k}^{r-1}[m] \widetilde{d}_{k}[r-1] e^{j \hat{\theta}_{k}[m]},
$$

and $\mathbf{b}[m]$ is of the form

$$
\mathbf{b}[m]=\left[\mathbf{a}_{1}^{r^{H}} \mathbf{c}_{1}^{r}[m] e^{j \hat{\theta}_{1}[m]} \ldots \mathbf{a}_{K}^{r^{H}} \mathbf{c}_{K}^{r}[m] e^{j \hat{\theta}_{K}[m]}\right]^{T}
$$

Given $\hat{\mathbf{d}}[r, m]$ and $\hat{\boldsymbol{\Theta}}[m]$, the error is also linear in the estimated channels as

$$
e[m]=v[m]-\mathbf{a}^{H} \hat{\mathbf{u}}_{\hat{\mathbf{\Theta}}}[m],
$$

where

$$
\hat{\mathbf{u}}_{\hat{\boldsymbol{\Theta}}}[m]=\left[\hat{\mathbf{u}}_{1}^{T}[m] e^{j \hat{\theta}_{1}[m]} \ldots \hat{\mathbf{u}}_{K}^{T}[m] e^{j \hat{\theta}_{K}[m]}\right]^{T} .
$$

Finally, the error is also linear in the vector $\hat{\boldsymbol{\Theta}}[m]$ given the symbol and channel estimates:

$$
e[m]=v[m]-\hat{\boldsymbol{\Theta}}^{T}[m] \mathbf{f}[m],
$$

where

$$
\mathbf{f}[m]=\left[\mathbf{a}_{1}^{H}[m] \hat{\mathbf{u}}_{1}[m] \ldots \mathbf{a}_{K}^{H}[m] \hat{\mathbf{u}}_{K}[m]\right]^{T} .
$$

When calculating $\hat{v}[m](6)$ and $\mathbf{f}[m]$ (14), a truncated version of the channel estimates $\left\{\mathbf{a}_{k}\right\}_{k=1}^{K}$ is used in order to reduce the estimation noise. All the taps below $G \cdot \max \left|\mathbf{a}_{k}\right|$ are set to zero. 
For each sample $m$ of the $r$-th symbol interval, the cyclic coordinate descent is applied (block labeled as 'update all the estimates' in Figure 3) as:

$$
\begin{gathered}
\hat{\mathbf{d}}[r, m+1]=\operatorname{RLS}(\hat{\mathbf{d}}[r, m], \mathbf{b}[m], e[m]) \\
\mathbf{a}[m+1]=\operatorname{NLMS}\left(\mathbf{a}[m], \hat{\mathbf{u}}_{\Theta}[m], e[m]\right) \\
\hat{\boldsymbol{\Theta}}[m+1]=\operatorname{DPLL}(\hat{\boldsymbol{\Theta}}[m], \mathbf{f}[m], e[m])
\end{gathered}
$$

where RLS is the Recursive Least Squares algorithm [5], NLMS is the Normalized Least Mean Squares algorithm [5], and DPLL stands for a second-order digital phase-locked loop [6]. When the last sample $\left(m=\operatorname{SF} N_{s c}(r+1)-1\right)$ of the block has been processed and used to update all the variables, the estimate $\hat{\mathbf{d}}[r, m]$ becomes the final estimate $\hat{\mathbf{d}}[r]$ of the symbols $\mathbf{d}[r]$. The final estimate can be then mapped into the signal space to obtain the decision $\widetilde{\mathbf{d}}[r]$. In the multichannel configuration of the proposed detector, decisions are made after the array processing.

During the training preamble, the operation of the detector is the same except for symbol estimation, which is not performed. Known values of the symbols are used to construct $\{\hat{\mathbf{u}}[m]\}_{k=1}^{K}$. This yields reliable estimates of channels and phases by the end of training. After training, each symbol is processed using the channel and phase estimates obtained from the previous block, while symbol estimates are reset to zero at the beginning of a block. The vector of symbol estimates can be viewed as an adaptive filter that has to acquire a different value for each block, starting from the initial value zero.

We consider constant-amplitude modulations where the information is contained in the phase of the symbols. If the value of SF is small, acquisition of the true symbols has to be performed fast. To do so, the forgetting factor of the RLS is kept high. As a consequence, the final estimates may have low amplitude. This problem is overcome by performing multiple passes over the same data block, where the initial values of the symbol estimates are the final estimates obtained in the last pass. We denote the number of passes (iterations) performed over the same data block by $N_{i t}$. After $N_{i t}$ passes, a final pass is performed as in training, using the obtained decisions instead of the true values in order to help the channel and phase estimates to keep their consistency along time.

\section{B. The Multichannel CCD Detector}

In a multichannel configuration, shown in Figure 4, the receiver uses as many single-channel $\mathrm{CCD}$ detectors as there are elements of the array $(Q)$. The MMSE combiner forms the final estimate of each user's symbol $\hat{d}_{k}$ (the index $r$ is dropped for the sake of simplicity) as a weighted sum of the individual estimates from all receiver elements.

The optimal combiner weights $\mathbf{t}_{k}$ are obtained by minimizing the metric

$$
\mathcal{J}_{\mathrm{MMSE}}=E\left\{\left|\widetilde{d}_{k}-\hat{d}_{k}\right|^{2}\right\} \quad ; \quad \hat{d}_{k}=\mathbf{t}_{k}^{H} \hat{\mathbf{d}}_{k}
$$

where $\mathbf{t}_{k} \in \mathbb{C}^{Q \times 1}$ is the combiner of the $k$-th user, $\widetilde{d}_{k}$ is the symbol decision obtained by mapping of $\hat{d}_{k}$ into the symbol

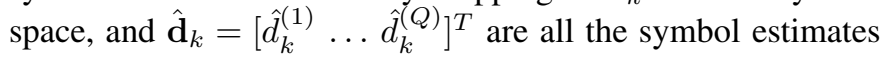

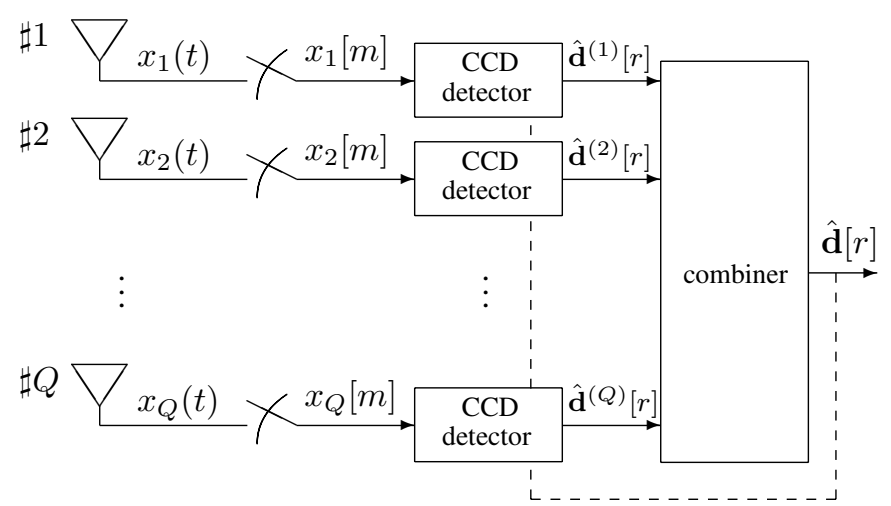

Fig. 4. Structure of the multichannel CCD detector.

of the $k$-th user. We assume that the $Q$ symbol estimates of user $k$ consist of the true value distorted by additive spatially uncorrelated noise, whose variance is estimated as

$$
\hat{\sigma}_{k, q}^{2}=\left|\widetilde{d}_{k}^{(q)}-\hat{d}_{k}^{(q)}\right|^{2} .
$$

Using the Wiener solution and the Woodbury's identity, we arrive at the following expression for the MMSE combiner of the $k$-th user

$$
\mathbf{t}_{k}=\frac{1}{1+\sum_{q=1}^{Q} 1 / \hat{\sigma}_{k, q}^{2}}\left[\hat{\sigma}_{k, 1}^{-2} \ldots \hat{\sigma}_{k, Q}^{-2}\right]^{T}
$$

and the final solution for the estimate $\hat{d}_{k, \text { MMSE }}$ of the symbol of the $k$-th user is

$$
\hat{d}_{k, \mathrm{MMSE}}=\mathbf{t}_{k}^{H} \mathbf{d}_{k}=\frac{1}{1+\sum_{q=1}^{Q} 1 / \hat{\sigma}_{k, q}^{2}} \sum_{q=1}^{Q} \frac{\hat{d}_{k}^{(q)}}{\hat{\sigma}_{k, q}^{2}}
$$

The symbol decisions formed using these estimates are fed back (dashed line of Figure 4) to the bank of single-channel CCD detectors and used to perform the last pass over the data block.

\section{EXPERIMENTAL RESULTS}

\section{A. Signal Design}

The signals were recorded during an experiment near Elba, Italy, in the fall of 2003. The DS-CDMA signals corresponding to four users were transmitted over a $2.3 \mathrm{~km}$ long shallow water channel (100 $\mathrm{m}$ depth). The signals were received over a 12 element array, submerged at $30 \mathrm{~m}$, with uniform element spacing of $0.15 \mathrm{~m}$.

Every user transmitted a packet of information up to 8 seconds long. The users were coarsely synchronized to within a symbol period. The modulation used was QPSK, and the chip rate was $16 \mathrm{kchips} / \mathrm{s}$. A carrier frequency of $35 \mathrm{kHz}$ and raised cosine pulses with roll-off factor $\alpha=0.25$ were used within the bandwidth 25-45 kHz. DS-CDMA was implemented using Kasami sequences of length 15, 63, and 255.

Each data packet consisted of a burst of $N_{t r}$ all-one training symbols followed by the information sequence. The corresponding data rate and other relevant parameters are listed in Table I. 
TABLE I

Data Rate and Packet Structure of each Configuration

\begin{tabular}{|c||c|c|c|c|c|}
\hline SF & $T$ & packet duration & $N_{t r}$ & $N_{d}$ & net data rate \\
\hline \hline 15 & $\sim 1 \mathrm{~ms}$ & $7.50 \mathrm{~s}$ & 320 & 7680 & $2048 \mathrm{bps} / \mathrm{user}$ \\
63 & $\sim 4 \mathrm{~ms}$ & $7.88 \mathrm{~s}$ & 80 & 1920 & $\sim 500 \mathrm{bps} / \mathrm{user}$ \\
255 & $\sim 16 \mathrm{~ms}$ & $7.97 \mathrm{~s}$ & 20 & 480 & $\sim 120 \mathrm{bps} / \mathrm{user}$ \\
\hline
\end{tabular}

\section{B. Example}

Here, we report on the $\mathrm{SF}=255$ case, which offers the best results. Detailed results of signal processing of all the configurations can be found in [7]. For the case of SF $=255,4$ receive elements sufficed to decode without errors the packets of all the users, resulting in a total throughput of $480 \mathrm{bps}$. Results of data processing are shown in Figures 5-7. Shown are the following parameters: scatter diagram of the data symbol estimates for the four users, estimated channel impulse responses (corresponding to each receive element), and phase estimates. The four users' signals are decoded with zero errors. Accurate channel estimates are obtained (Figure 6), and the Doppler shifts of a few Hertz due to drift are correctly tracked (Figure 7).
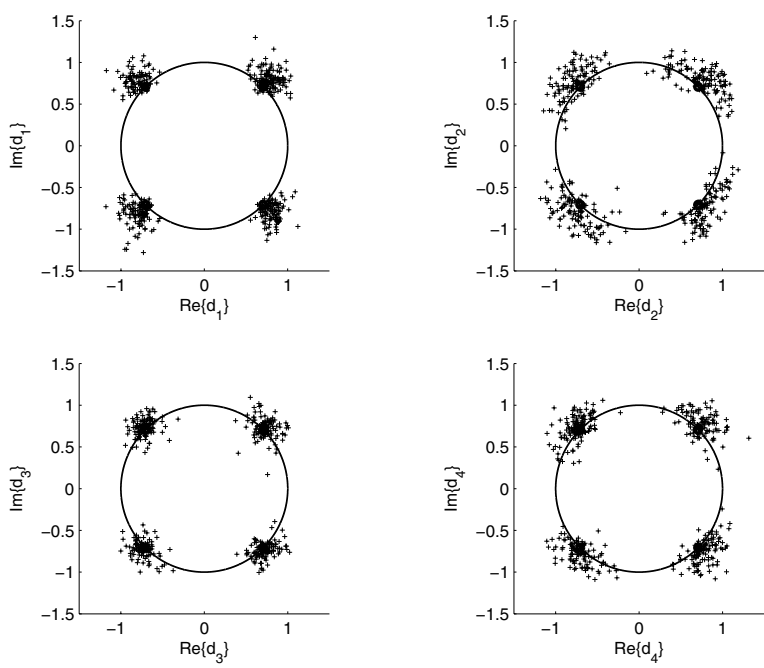

Fig. 5. Scatter diagrams for $\mathrm{SF}=255,4$ users, 4 receive elements.

\section{CONCLUSIONS}

The multi-user detector proposed in this paper is based on joint data detection and channel estimation. Assuming that the data symbols are available, they are used to estimate the channel responses, which, in turn, are used to refine the symbol estimates, and so on. The receiver was implemented in a multichannel configuration, which provides the array processing gain necessary for many of the underwater acoustic channels. The complexity of the algorithm is linear in the number of receive elements and it does not depend on the modulation level. The algorithm performance was demonstrated in a fouruser DS-CDMA configuration using experimental data. The quality of the detection algorithm was found to improve with the spreading factor SF, as the symbol estimation algorithm
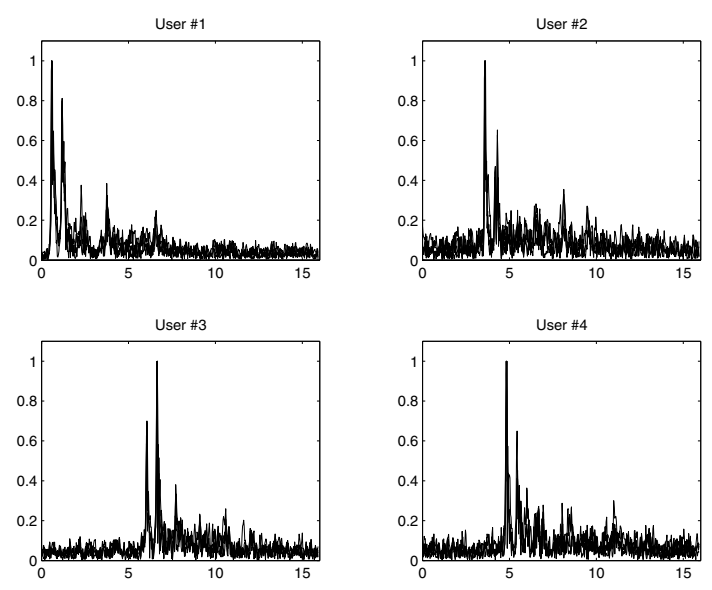

Fig. 6. Normalized channel impulse responses for SF $=255,4$ users, 4 receive elements. The horizontal axis, in milliseconds, spans one symbol duration.
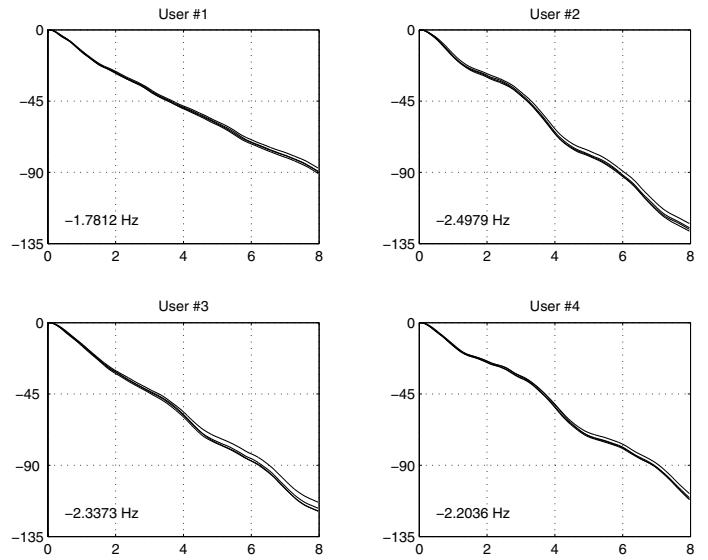

Fig. 7. Phase offsets in [RAD] and Doppler shifts in $[\mathrm{Hz}]$ for $\mathrm{SF}=255$, 4 users, 4 receive elements. The horizontal axis, in seconds, spans the packet duration.

has more time to reach its final solution. Future work in this area should concentrate on algorithm refinement to enable its operation with lower spreading factors.

\section{REFERENCES}

[1] J.G. Proakis and M. Salehi, Communication systems engineering, Prentice Hall, 1994.

[2] S. Verdú, Multiuser detection, Cambridge University Press, 1998.

[3] J. Luo, G. Levchuk, K. Pattipati, and P.Willett, "A class of coordinate descent methods for multiuser detection", in Proc. IEEE ICASSPOO Conference, June 2000.

[4] F. Hasegawa, J. Luo, K.. Pattipati, P. Willett, and D. Pham, "Speed and accuracy comparison of techniques for multiuser detection in synchronous CDMA", IEEE Trans. on Communications, vol. 52, pp. 540-545, April 2004.

[5] S. Haykin, Adaptive filter theory, Prentice Hall, 2001.

[6] M. Stojanovic and Z. Zvonar, "Multichannel processing of broad-band multiuser communication signals in shallow water acoustic channels", in IEEE Trans. on Oceanic Engineering, vol. 21, pp. 156-166, April 1996.

[7] E. Calvo, Multiuser Detection for Underwater Acoustic DSCDMA Systems, M.S. Thesis, Technical University of Catalonia / Massachusetts Institute of Technology, Aug. 2004. 